\title{
Preclinical evaluation of a new robot- assisted surgical system for use in gynecology minimal access surgery
}

\author{
Marcus Carey ${ }^{1}$, Anish Bali, Ivilina Pandeva ${ }^{3}$, Ashish Pradhan ${ }^{4}$ and Mark Slack ${ }^{5^{*}}$
}

\begin{abstract}
Robot-assisted laparoscopy has been developed to overcome some of the important limitations of conventional laparoscopy. In particular, the provision of stable magnified three-dimensional vision, tremor filtering, motion scaling, and articulated instruments with robot-assisted surgery has the potential to enable more surgeons to perform more complex surgery compared with conventional laparoscopy. The aim of this study was to evaluate the utility of a new robot-assisted surgical system (Versius, CMR Surgical, Cambridge, UK) for gynecologic procedures in a preclinical setting. Cadaveric sessions were conducted to evaluate the ability of the system to complete all surgical steps required for a robot-assisted total laparoscopy hysterectomy. A live animal (porcine) model was used to assess the system in performing oviduct removal as a surrogate for robot-assisted total laparoscopy hysterectomy. Procedures were performed by experienced gynecologic surgeons, supported by a surgical team. The precise surgical steps conducted to conclude that the procedures could be fully completed were systematically recorded, as well as instruments used and endoscope angle. In total, six gynecologic procedures were performed in cadavers by four surgeons; 16/17 procedures were completed successfully. Positioning of the ports and bedside units reflected the surgeons' preferred laparoscopic setup and enabled good surgical access and reach, as exemplified by the high procedure completion rate. Oviduct removal procedures performed in pigs were all completed successfully by a single surgeon. This preclinical study of a new robot-assisted surgical system for gynecologic procedures demonstrated the safety and effectiveness of the system in cadaver and porcine models. Further studies are required to assess its clinical utility.
\end{abstract}

Keywords: Minimally invasive surgical procedures, Robotic surgical procedures, Gynecologic Surgical procedures, Hysterectomy

\section{Introduction}

Over the last 25 years, minimal access surgery (MAS) has become an essential component of modern gynecology surgery, with many laparoscopic techniques now considered routine [1]. Gynecologic MAS is associated with a number of advantages over open surgery, including reduced blood loss, fewer perioperative complications, reduced post-operative pain, earlier

\footnotetext{
* Correspondence: mark.slack@cmrsurgical.com

${ }^{5}$ CMR Surgical Ltd, 1 Evolution Business Park, Milton Road, Cambridge CB24 9NG, UK

Full list of author information is available at the end of the article
}

recovery, shortened hospital stay, and improved cosmesis [1-3]. However, MAS for complex surgery is technically demanding and associated with a steep learning curve $[1,4-7]$. This is due to certain limitations of conventional laparoscopy such as restricted movement, difficulty performing accurate suturing, uncomfortable positions for the surgeon and assistant, and twodimensional vision $[1-3,8]$. These limitations may contribute to the relatively low adoption of MAS for commonly performed gynecologic procedures, such as hysterectomy and sacrocolpopexy [4, 9-11].

\section{Springer Open}

(๑) The Author(s). 2020 Open Access This article is licensed under a Creative Commons Attribution 4.0 International License, which permits use, sharing, adaptation, distribution and reproduction in any medium or format, as long as you give appropriate credit to the original author(s) and the source, provide a link to the Creative Commons licence, and indicate if changes were made. The images or other third party material in this article are included in the article's Creative Commons licence, unless indicated otherwise in a credit line to the material. If material is not included in the article's Creative Commons licence and your intended use is not permitted by statutory regulation or exceeds the permitted use, you will need to obtain permission directly from the copyright holder. To view a copy of this licence, visit http://creativecommons.org/licenses/by/4.0/. 
Robot-assisted laparoscopy has made progress in overcoming some of the important shortcomings of conventional laparoscopy by providing an ergonomic operating position, a stable magnified three-dimensional view, tremor filtering, motion scaling, and articulated or wristed instruments, allowing for precise tissue dissection and suturing $[12,13]$. By shortening the learning curve, these advantages may enable more surgeons to perform complex MAS procedures, and could extend the feasibility of MAS to a greater body of surgeons [2]. In addition, robot-assisted MAS could be feasible in a wider range of patients, such as those with a higher body mass index (BMI) $[14,15]$.

The new robot-assisted surgical system (Versius, CMR Surgical, Cambridge, UK; Supplementary Figure 1) used in this study was developed to aid surgeons in performing MAS. The system has been designed to mimic the articulation of the human arm, and the wristed instrument tip provides seven degrees of freedom inside the patient, with the purpose of improving surgical access compared with standard laparoscopic surgery. The surgeon interacts with the system through hand controllers, with feedback provided on the system's open surgeon console, which allows the surgeon to sit or stand during surgery. The console's head-up display provides a threedimensional video from the endoscopic camera together with a display overlay. The bedside team view a twodimensional version of the endoscope feed and display overlay via an auxiliary display on the visualisation bedside unit (BSU), and are able to access controls and feedback on up to four instrument BSUs. The BSUs are small enough to be used in a standard operating room (OR) and moved easily within a single OR and between ORs [16].

The IDEAL-D (Idea, Development, Exploration, Assessment, Long-term study for medical devices) framework provides recommendations for improving the evidence base from research at each stage of surgical innovation [17]. The operational safety and ease of use of the system was validated previously in human cadaver studies [18]. Consistent with the IDEAL-D framework, the next step in evaluating the system's suitability for use in gynecology surgery is preclinical evaluation [17, 19]. The preclinical studies described here had two aims: (1) demonstrate the ability of the system to perform several gynecologic procedures using cadavers and (2) assess the system's ability to perform oviduct removal, as a surrogate for robot-assisted total laparoscopic hysterectomy (RALH) in a live animal (porcine) model.

\section{Materials and methods}

\section{Study design}

This was an observational study with cadaver and live animal surgery components. The main purpose of the cadaver component was to assess the system's capabilities in a variety of gynecologic procedures. The main aims of the live animal component were to evaluate surgical complications and post-operative recovery. Cadaver studies were undertaken at the Evelyn Cambridge Surgical Training Centre, UK, between 26 July 2018 and 16 April 2019. All cadavers were donated with consent. The live animal porcine study was undertaken at Covance CRS Ltd (formerly Envigo Ltd), Huntingdon, UK between 12 and 24 October 2018. This study was designed to align with the principles of the 3Rs (replacement, reduction and refinement) and was conducted in accordance with current, internationally recognised Good Laboratory Practice Standards and the UK Animals (Scientific Procedures) Act 1986, Amendment Regulations 2012. All procedures were performed in a simulated operating room in order to mimic clinical practice.

\section{Surgical team}

Procedures were performed by a surgeon supported by a surgical team. The surgeon performed the surgical steps for the procedure and evaluated the system. The assistant surgeons carried out any additional manual tasks as instructed by the surgeon. Trained observers recorded port and BSU placements, intra-operative events, and outcomes.

The four surgeons who performed the procedures on cadavers were accredited, practicing high-volume gynecologic surgeons as defined by $>50$ complex laparoscopic procedures/annum. The surgeon performing procedures in pigs was also a practicing surgeon, certified in good laboratory practice (GLP) and possessed UK Home Office licenses. All users were trained to use the robot-assisted surgical system, and three of the four surgeons had experience performing procedures using the system in prior studies [18]. During the procedures described here, a professional CMR Surgical education team also provided expert advice at the console.

\section{Cadaver studies}

A variety of gynecologic procedures were performed on 11 female cadavers which had not undergone previous abdominal or pelvic surgery. Cadavers were selected to represent a spectrum of BMIs to reflect the wide range in size and shape of human anatomy.

Each surgeon determined the port and BSU positions, based on their established, standard technique of performing the same procedure by conventional laparoscopy. Instrument and accessory ports were inserted into the abdominal cavity after establishing pneumoperitoneum using a 14 gauge disposable Veress insufflation needle. Port and BSU positions were recorded using a $20-\mathrm{cm}$ grid (covering $320 \mathrm{~cm} \times 320 \mathrm{~cm}$ ) laid out on the OR floor (Fig. 1); BSU positions in relation to anatomical 


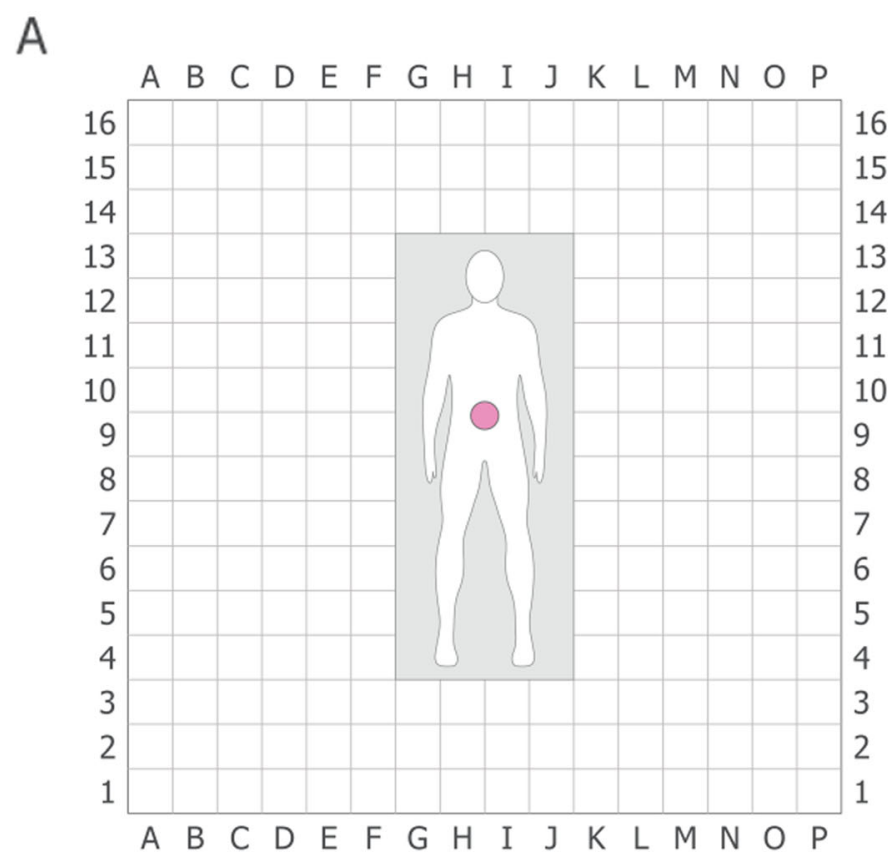

B

Fig. 1 Record keeping of bedside unit positions. a Outline of the grid template used to measure BSU location. To ensure reliable and standardized reporting of measurements, a grid of $20 \mathrm{~cm} \times 20 \mathrm{~cm}$ squares was laid out on the OR floor such that the overall grid was $320 \mathrm{~cm} \times$ $320 \mathrm{~cm}$. b Example OR setup with grid. BSU, bedside unit; OR, operating room

land marks on the cadaver were also recorded. Port and BSU positions were altered from one procedure to the next in response to difficulties in surgical access and reach such as inability to reach surgical site, instruments too close to surgical site, and arms clashing due to port positioning. Positions were deemed suitable if good access to the surgical site(s) was achieved without arm clashing and there was a minimal need to reposition BSUs. The precise surgical steps conducted to make the assessment that the procedures could be fully completed were recorded, as well as instruments used (including manual laparoscopic instruments) and endoscope angle.

\section{Porcine study}

Six large white hybrid domestic female pigs aged 18-20 weeks with a median weight of $42.0 \mathrm{~kg}$ (range 37.5-44.5 $\mathrm{kg}$ ) underwent oviduct removal as a surrogate for RALH. Prior to the procedure and in accordance with GLP in animal studies, each pig was sedated before transfer to the OR, where the animal was placed under general anesthesia and intubated. Instrument and accessory ports were inserted into the abdominal cavity in insufflated bodies using a 14 Gauge disposable Veress insufflation needle. Local anesthesia was applied to the intended port positions; ports were placed by the surgeon according to a predetermined protocol.

Table 1 Summary of procedures performed and successful completion in cadavers

\begin{tabular}{|c|c|c|c|c|}
\hline Procedure & Number performed & Number successfully completed & Number of surgeons ${ }^{*}$ & $\begin{array}{l}\text { Number of unique port } \\
\text { configurations }\end{array}$ \\
\hline Burch colposuspension & 3 & 3 & 2 & 3 \\
\hline Para-vaginal wall repair & 1 & 1 & 1 & 1 \\
\hline Sacrocolpopexy & 1 & 1 & 1 & 1 \\
\hline Sacrohysteropexy & 3 & 3 & 1 & 2 \\
\hline $\begin{array}{l}\text { Sub-total laparoscopic hysterectomy } \\
\text { with sacrocervicopexy }\end{array}$ & 3 & 3 & 3 & 3 \\
\hline $\begin{array}{l}\text { Robot-assisted total laparoscopic } \\
\text { hysterectomy (RALH) }\end{array}$ & 6 & 5 & 2 & 4 \\
\hline Total & 17 & $16(94.1 \%)$ & - & 14 \\
\hline
\end{tabular}

${ }^{\mathrm{a}}$ One procedure could not be completed due to the physical condition of the cadaver

*Total number of surgeons who completed a given procedure; individual cases were completed with one surgeon 
During the procedure, intra-operative blood loss was measured, and intra-operative adverse events were recorded. Pigs were divided into two groups: non-recovery and recovery. Non-recovery pigs were euthanized without recovery from anesthesia with pentobarbitone. Successful and safe procedure completion was confirmed in non-recovery pigs before the procedure was attempted in recovery pigs. In recovery pigs, wounds were closed, anesthesia was discontinued, and animals were observed for signs of ill health or changes in behavior and/or activity. Post-operative analgesia, antibiotic treatment, and other treatments as appropriate were administered by a

\section{A \\ $11 \mathrm{~mm}$ endoscope port \\ $11 \mathrm{~mm}$ accessory port \\ $5 \mathrm{~mm}$ instrument and accessory port \\ $5 \mathrm{~mm}$ instrument port}

\section{Surgeon 1}

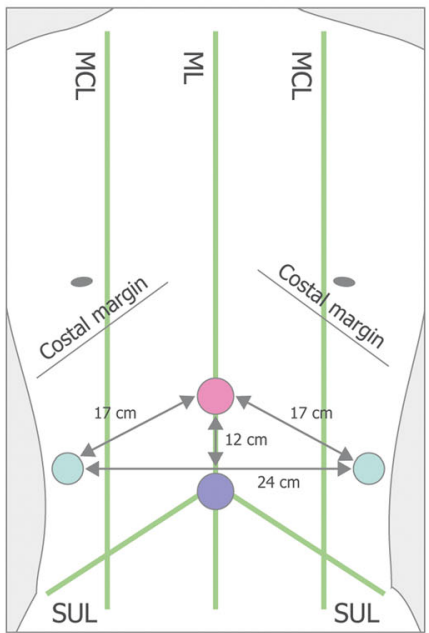

Surgeon 2

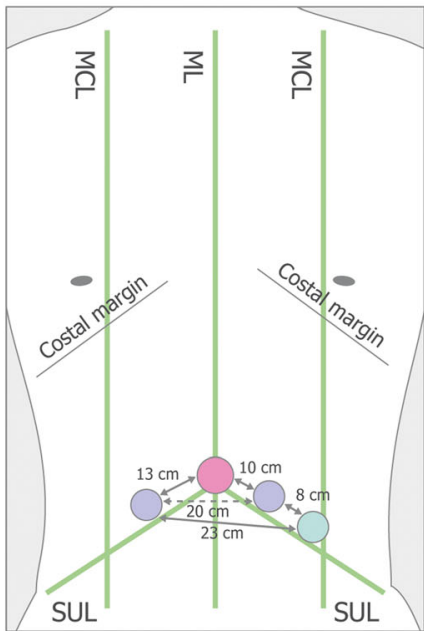

B

\section{Surgeon 1}

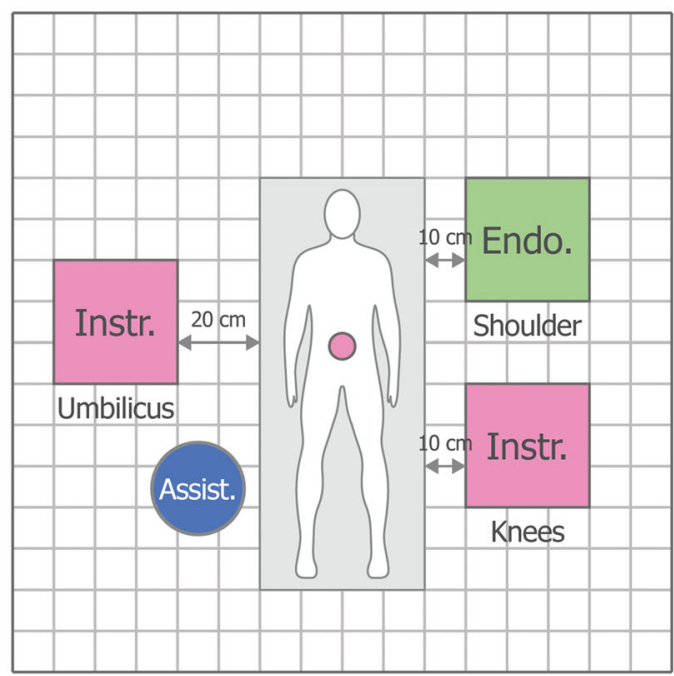

Surgeon 2

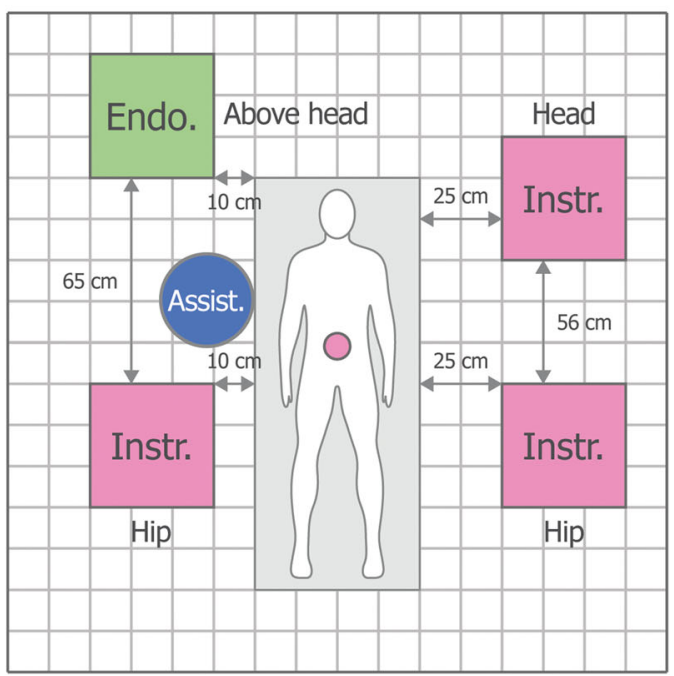

Fig. 2 Common port and bedside unit positions tested in cadaver studies. a Common port positions for RALH. The endoscope angle was $0^{\circ}$ for all procedures. Ports were generally organized in a triangular configuration. The surgeons placed either an accessory port (Surgeon 1) or an endoscope port (Surgeon 2) at the umbilicus (where the ML crosses the SUL); both surgeons placed instrument ports on both sides of the umbilicus. For Surgeon 2's configuration, the left and middle accessory ports were sometimes also used as instrument ports. $\mathbf{b}$ The modular system facilitated flexibility in the positioning of the BSU and surgical assistant; the illustrated positions reflect the most common surgical setup for each operating surgeon. Superimposed rectangle represents the surgical table with measurements detailing the distance between instrument BSUs and the surgical table or other BSUs. Diagrams are not drawn to scale. Pink dot indicates the umbilicus. Assist, surgical assistant; BSU, bedside unit; Endo, endoscope; Instr, instrument; MCL, midclavicular line; ML, midline; RALH, robot-assisted total laparoscopic hysterectomy; SUL, supine-umbilical line 
veterinary surgeon. Pigs were euthanized after 22-29 days and subject to a detailed necropsy, with specific reference to surgical sites and assessment of successful organ removal.

\section{Results}

\section{Procedure completion in cadavers}

The cadavers represented a wide range of BMIs; median BMI was $22.31 \mathrm{~kg} / \mathrm{m}^{2}$ (range $14.5-33.0 \mathrm{~kg} / \mathrm{m}^{2}$ ). In total, six types of gynecologic procedures were performed. The main gynecologic procedure evaluated was RALH (six procedures performed by two surgeons). Burch colposuspension, paravaginal wall repair, sacrocolpopexy, sacrohysteropexy, and sub-total laparoscopic hysterectomy with sacrocervicopexy were also performed to demonstrate the ability of the system to complete complex gynecologic procedures particularly where suturing is involved. A total of 17 procedures were performed, of which 16 (94.1\%) were successfully completed. One RALH could not be completed due to the poor physical condition of the cadaver (Table 1).

\section{Common port and BSU positions for RALH in cadavers}

RALH was selected to be the main procedure for evaluation because hysterectomy is one of the most common gynecology surgeries performed and exemplifies the difficulties of achieving good access and challenging suturing of the vaginal cuff. Robotic assistance has been considered helpful in overcoming these issues $[1,4,9,10]$.

For RALH, the cadaver was placed in lithotomy to allow abdominal and vaginal access. Steep Trendelenburg position was used for all procedures. Two surgeons performed three procedures each. Figure $2 \mathrm{a}$ illustrates the two different port placement configurations used by each of the two surgeons. Two different BSU configurations were used, indicative of the different port placements used by each surgeon (Fig. 2b): (1) the visualisation BSU was located around the left shoulder, and two instrument BSUs were positioned on both sides of the operating table (Fig. 3); (2) the visualisation BSU was located above the head on the right side, and three instrument BSUs were used, one by each knee and a third by the head on the left side.

The port and BSU configurations used were all found to be suitable for successful procedure completion, which encompassed mobilization of the uterus, dissection of the parametria, extraction of the uterus, and closure of the vaginal vault (Table 2). Tissue manipulation, dissection, and suturing for the key surgical steps were achieved using the system's monopolar hook, bipolar Maryland grasper, curved scissors, fenestrated grasper, and needle holder. Manual graspers and manual laparoscopic scissors were also used.

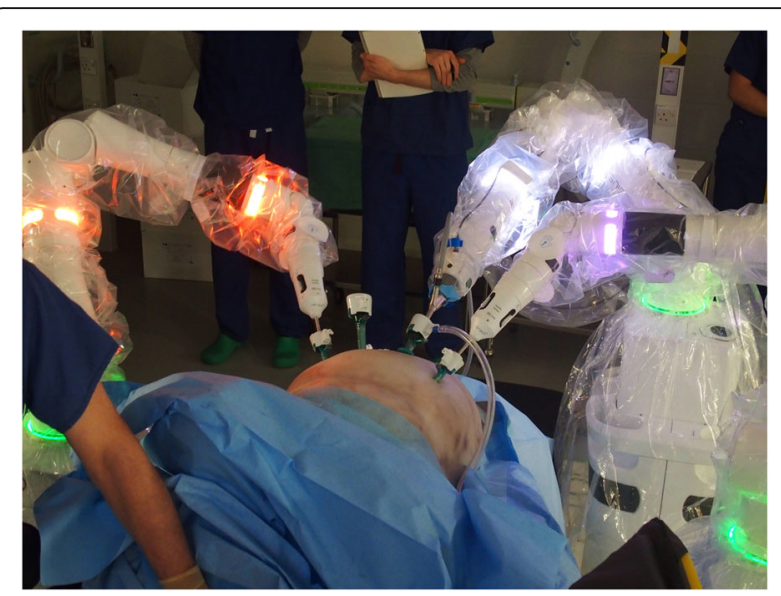

Fig. 3 Physical arrangement of the new robot-assisted surgical system. Image of the physical arrangement of the new robotassisted MAS system setup during RALH, corresponding to the left panel shown in Fig. 2b. Visualisation BSU in white and instrument BSUs in orange and green. BSU, bedside unit; MAS, minimal access surgery; RALH, robot-assisted total laparoscopic hysterectomy

\section{Safety in live animals}

Six oviduct removals (non-recovery $n=2$, recovery $n=$ 4) were performed in pigs as a surrogate for RALH. All procedures were successfully completed. There was one device-related intra-operative complication in one nonrecovery pig: evidence of thermal injury to the bowel from the monopolar instrument shaft. Only two nondevice-related intra-operative complications were recorded (one related to port insertion and one related to replacement of a port that was removed too early). Intra-operative blood loss was recorded as $\sim 10 \mathrm{~mL}(n=$ $1)$ or none $(n=5)$. Clinical observations of the recovery pigs post-operatively revealed no signs of ill health or distress, and all recovery pigs gained weight postsurgery. Overall, recovery pigs remained in good health

Table 2 Surgical steps in robot-assisted total laparoscopic hysterectomy

\section{Surgical steps}

- Lift bowel out of pelvis

- Grasp right round ligament and pull uterus to the left

- Divide the right round ligament

- Divide the right infundibular ligament

- Open anterior leaf of broad ligament

- Open posterior leaf of broad ligament

- Skeletonize the uterine artery

- Grasp the left round ligament

- Pull the uterus to the right

- Divide the left round ligament

- Divide the left infundibular ligament

- Skeletonize the left broad ligament

- Incise and dissect the parametria on both sides

- Open the posterior vaginal cuff

- Open the anterior vaginal cuff

- Complete colpotomy and remove uterus

- Close vault with interrupted sutures 
throughout the post-operative recovery period. At macroscopic post-mortem examination, assessments revealed minor signs of inflammation around one port site, and cysts presented on top of the vaginal cuff in two of four pigs. In one pig, when the cyst was removed, the vaginal cuff was noticed to be open (Fig. 4). In all pigs, surrounding organs appeared healthy with no signs of injury or inflammation.

\section{Discussion}

The cadaver studies demonstrated that the new robotassisted MAS system can be used for robotic surgery in a variety of gynecologic procedures. The system's flexibility enabled adequate surgical access and reach in the pelvis, even in specimens with a high BMI. The instrument articulation, ergonomic handgrip, and console allowed surgeons to successfully complete these procedures within the confines of the pelvis. The procedures described were performed in a manner reflecting how they would be performed in the clinical setting, from surgical setup to the surgical steps performed. Moreover, the ability to perform oviduct removal safely and effectively has been demonstrated in a live animal model, providing a good simulation of system performance expected in live humans.

The port placement for RALH with current robotic systems usually requires two or three instrument ports, one endoscope port, and one accessory port [20]. Therefore, an operating surgeon is often limited to a narrow range of port configurations. Findings from this study demonstrate that a variety of port placements provide adequate surgical access and reach; this flexibility enabled surgeons to effectively transfer their preferred laparoscopic port placements for use with the robotic system.

\section{Further development of the system}

The system tested in these studies was not the final design. Incremental changes to instruments, hardware, and software were made throughout these studies to improve the design of the system and the surgical setup for each type of procedure tested (e.g., repositioning BSUs after an arm clash to mitigate the risk of a reoccurrence). Further studies will be performed to more quantitatively assess instrument functionality, particularly that of the electrosurgical instruments, to improve their safety and mitigate the risk of tissue burning. Procedures that have been performed a limited number of times in cadavers will be repeated to further optimize the use of the new robot-assisted MAS system for these surgeries. The aim is to ensure the robot and its use are perfected ahead of clinical studies in gynecology surgery.

\section{Limitations}

Cadaver and porcine models are frequently used in surgical training, and each model has advantages and disadvantages in terms of its ability to test robotic surgical ability in live humans. Porcine models bear greater similarity to live tissue handling, dissection, surgical plane identification, and control of bleeding in live human surgery. However, cadavers provide much greater anatomical relevance and realism to live humans than pigs [21]. Testing the new system in both cadaver and pig models balances the bias introduced by each model; however, it is impossible to completely replicate the experience and performance of the robot for surgery in live humans. Although only oviduct removal was performed in pigs, this procedure was selected to provide a good simulation of system performance for gynecologic procedures in live humans. The number of procedures performed was deemed suitable to generate sufficient evidence for the safety of the new robot-assisted MAS system whilst aligning with the 3 Rs.

\section{Conclusion}

The studies presented here cover the preclinical assessment of Versius for gynecologic procedures in cadaveric and porcine models. Several types of gynecology surgeries were tested in cadavers, with the surgeons evaluating
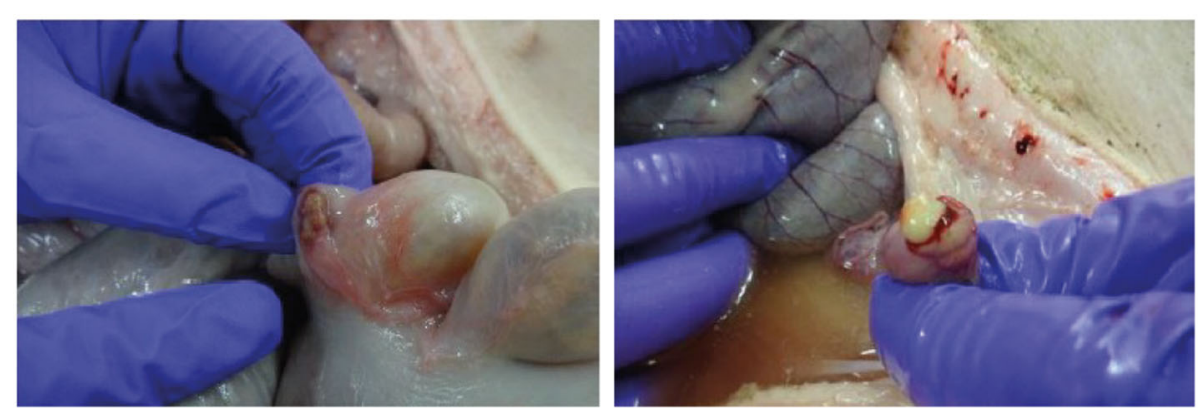

Fig. 4 Necropsy findings from recovery pigs. Necropsy of oviduct removal from a recovery pig showing good healing of the vaginal cuff (left panel). Necropsy of oviduct removal from a recovery pig showing the presence of a cyst on the vaginal cuff (right panel) 
a range of port and BSU positions; all but one procedure was successful. Oviduct removal was also performed safely and effectively in a live animal model. These results support the progression to assessment of the new robot-assisted MAS system in clinical studies of gynecology surgery as per the IDEAL-D framework [17].

\section{Supplementary information}

Supplementary information accompanies this paper at https://doi.org/10. 1186/s10397-020-01069-0.

Additional file 1: Figure S1. Schematic overview of the Versius system

\section{Abbreviations}

3Rs: Replacement, reduction, and refinement; BMl: Body mass index; BSU: Bedside unit; GLP: Good laboratory practice; IDEAL-D: Idea, Development, Exploration, Assessment, Long-term study for medical devices; MAS: Minimal access surgery; OR: Operating room; RALH: Robot-assisted total laparoscopic hysterectomy

\section{Acknowledgements}

The authors thank the individuals who donated their bodies to medical research and also thank surgeon Mr. Jonathan Lippiatt, MBBCh, Division of Oncologic Gynecology, Department of Obstetrics \& Gynecology, Poole Hospital NHS Foundation Trust, Poole, Dorset, UK, as well as the investigators and their teams who took part in this study. The authors also acknowledge Marc Lynch, PhD, Vivian Peirce, PhD, and Simon Foulcer, PhD, from Costello Medical, UK, for medical writing and editorial assistance based on the authors' input and direction.

\section{Authors' contributions}

Substantial contributions to study conception and design: $A B, M C, I P, A P$, and MS; substantial contributions to analysis and interpretation of the data: $A B, M C, I P, A P$, and $M S$; drafting the article or revising it critically for important intellectual content: $A B, M C, I P, A P$, and $M S$; final approval of the version of the article to be published: $A B, M C, I P, A P$, and $M S$.

\section{Funding}

This study was sponsored by CMR Surgical. This article was based on the original studies TR-0732, TR-0883, TR-0913, and TR-1351 sponsored by CMR Surgical. Support for third-party writing assistance for this article was funded by CMR Surgical in accordance with Good Publication Practice (GPP3) guidelines (http://www.ismpp.org/gpp3)

\section{Availability of data and materials}

All data generated or analyzed during this study are included in this published article [and its supplementary information files].

\section{Ethics approval and consent to participate}

All cadaver studies were conducted at The Evelyn Cambridge Surgical Training Centre, Back Lane, Melbourn, Hertfordshire, SG8 6DP, UK. The Evelyn Centre is certified as Health Tissue Authority (HTA) compliant under license number 12603. The HTA designated individual responsible at the facility is Mr Christopher Constant MA (Cantab) LLM MCh FRCS RMIMI. All studies conducted by CMR Surgical at The Evelyn Centre met the required HTA, health and safety, and ethical considerations relating to the use of donated cadaveric tissue in dissection, teaching, research, and development. Porcine work was conducted in accordance with the applicable sections of the United Kingdom Animals (Scientific Procedures) Act 1986, Amendment Regulations 2012 (the Act) and in compliance with the requirements of current, internationally recognized Good Laboratory Practice Standards (UK Good Laboratory Practice Regulations; Statutory Instrument 199 No. 3106, as amended by Statutory Instrument 2004 No. 994, OECD Principles of Good Laboratory Practice ENV/MC/CHEM(98)17, and EC Commission Directive 2004/10/EC) and was designed to align with the principles of the 3Rs (replacement, reduction, and refinement).

\section{Consent for publication}

Not applicable.

\section{Competing interests}

$A B$ and $A P$ are on the medical advisory board and are shareholders of CMR surgical. IP has received consultancy fees from CMR surgical. MC has no financial interest in CMR. However, CMR Surgical covered the cost of travel and accommodation to and from Melbourne, Australia, to allow MC to undertake the cadaver surgery. He received no honorarium. MS is the Chief Medical Officer and co-founder of CMR Surgical.

\section{Author details}

${ }^{1}$ Department of Urogynecology, Royal Women's Hospital, Parkville, Australia.

${ }^{2}$ University Hospitals of Derby and Burton NHS Foundation Trust, Royal Derby Hospital, Derby, UK. ${ }^{3}$ Department of Urogynecology, Cambridge University Hosptals NHS Trust, Addenbrooke's Hospital, Cambridge, UK. ${ }^{4}$ Department of Urogynecology, Cambridge University Hospitals NHS Foundation Trust, Addenbrooke's Hospital, Cambridge, UK. ${ }^{5}$ CMR Surgical Ltd

1 Evolution Business Park, Milton Road, Cambridge CB24 9NG, UK.

Received: 16 January 2020 Accepted: 30 March 2020

Published online: 14 April 2020

\section{References}

1. Powell F, Khaund A (2016) Laparoscopy and laparoscopic surgery. Obstetrics, Gynaecology \& Reproductive Medicine 26:297-303

2. Lawrie TA, Liu H, Lu D et al (2019) Robot-assisted surgery in gynaecology. Cochrane Database Syst Rev

3. Lee J, Asher V, Nair A et al (2018) Comparing the experience of enhanced recovery programme for gynaecological patients undergoing laparoscopic versus open gynaecological surgery: a prospective study. Perioperative Medicine 7:15

4. Nieboer TE, Spaanderman ME, Bongers MY et al (2010) Gynaecologists estimate and experience laparoscopic hysterectomy as more difficult compared with abdominal hysterectomy. Gynecol Surg 7:359-363

5. Jauffret C, Lambaudie E, Esterni B et al (2016) Learning curve of robotassisted laparoscopy in gynecologic oncology surgery: team training and impact on morbidity. Clin Surg 1:1112

6. Cela V, Freschi L, Simi G et al (2013) Robotic single-site hysterectomy: feasibility, learning curve and surgical outcome. Surg Endosc 27:2638-2643

7. Cela V, Marrucci E, Angioni S et al (2018) Robot-assisted laparoscopic singlesite hysterectomy: our experience and multicentric comparison with singleport laparoscopy. Minerva Ginecol 70:621-628

8. Claerhout F, Verguts J, Werbrouck E et al (2014) Analysis of the learning process for laparoscopic sacrocolpopexy: identification of challenging steps. Int Urogynecol J 25:1185-1191

9. Fuchs Weizman N, Maurer R, Einarsson Jl et al (2015) Survey on barriers to adoption of laparoscopic surgery. Journal of surgical education 72:985-994

10. Sinha R, Swarnasree G, Rupa B et al (2019) Laparoscopic hysterectomy for large uteri: outcomes and techniques. J Minim Access Surg 15:8-13

11. Haya N, Feiner B, Baessler K, et al (2018) Perioperative interventions in pelvic organ prolapse surgery. Cochrane Database of Systematic Reviews: CD013105

12. Bouquet de Joliniere J, Librino A, Dubuisson J-B, et al (2016) Robotic surgery in gynecology. Frontiers in Surgery 3: eCollection 2016

13. Orady M, Hrynewych A, Nawfal AK et al (2012) Comparison of roboticassisted hysterectomy to other minimally invasive approaches. JSLS: Journal of the Society of Laparoendoscopic Surgeons 16:542

14. Corrado G, Mereu L, Bogliolo S, et al (2019) Comparison between single-site and multiport robot-assisted hysterectomy in obese patients with endometrial cancer: an Italian multi-institutional study. The international journal of medical robotics + computer assisted surgery : MRCAS: e2066

15. Buderath P, Aktas B, Heubner M et al (2015) Robot-assisted hysterectomy: a critical evaluation. Robot Surg Res Rev 2:51-58

16. Hares $L$, Roberts $P$, Marshall K et al (2019) Using end-user feedback to optimize the design of the Versius Surgical System, a new robot-assisted device for use in minimal access surgery. BMJ Surgery, Interventions \& Health Technologies 1:e000019

17. McCulloch P, Altman DG, Campbell WB et al (2009) No surgical innovation without evaluation: the IDEAL recommendations. Lancet 374:1105-1112 
18. Haig F, Chitty K, Medeiros A, et al (2020) Usability assessment of Versius, a minimally invasive robot-assisted surgical device. BMJ Surgery, Interventions \& Health Technologies: In press

19. Hirst A, Philippou Y, Blazeby J et al (2019) No Surgical innovation without evaluation: evolution and further development of the IDEAL framework and recommendations. Ann Surg 269:211-220

20. Nair R, Killicoat $K$, Ind TE (2016) Robotic surgery in gynaecology. The Obstetrician \& Gynaecologist 18:221-229

21. Stefanidis D, Yonce TC, Green JM et al (2013) Cadavers versus pigs: which are better for procedural training of surgery residents outside the OR? Surgery 154:34-37

\section{Publisher's Note}

Springer Nature remains neutral with regard to jurisdictional claims in published maps and institutional affiliations.

\section{Submit your manuscript to a SpringerOpen ${ }^{\circ}$ journal and benefit from:}

- Convenient online submission

- Rigorous peer review

- Open access: articles freely available online

- High visibility within the field

- Retaining the copyright to your article 